\title{
Index of New Taxa
}

\section{Edited by Riccardo M. Baldini (Editor in Chief)}

The new taxa, new combinations, new synonyms and the names described in volume 75 (2020) are listed below. The last number shows the page/s of publication and the asterisk means that the taxon is provided by an illustration or photo.

Vol. 75(1). 2020

Nepenthes latiffiana M.N. Faizal, A. Amin \& N. Dome, sp. nov. 75(1). 2020. p. $7^{\star}$

Nepenthes domei M.N. Faizal, A. Amin \& A. Latiff., sp. nov. ... 75(1). 2020. p. $10^{*}$

Isoetes xkarenae D.F. Brunton \& P.C. Sokoloff, hyb. nov. 75(1). 2020. p. $39-40^{*}$

Isoetes xfernaldii D.F. Brunton \& P.C. Sokoloff, hyb. nov. 75(1). 2020. p. $44-45$

Pedicellarum M. Hotta, syn. nov. 75(1). 2020. p. 91

Pothos degenerans S.Y. Wong \& P.C. Boyce \& A. Hay, sp. nov. 75(1). 2020. p. $91-93^{\star}$

Pothos ecclesiae P.C. Boyce, S.Y. Wong \& A. Hay, sp. nov 75(1). 2020. p. $93-96^{*}$

Pothos fractiflexus J. Joling, Pereira \& A. Damit, sp. nov. 75(2). 2020. p. $96-98^{*}$

Pothos paiei (M. Hotta) S.Y. Wong, A. Hay \& P.C. Boyce, comb. nov............................................. 75(1). 2020. p. 100*

Schismatoglottis imbakensis Kartini, S.Y. Wong \& P.C. Boyce, sp. nov. 75(1). 2020. p. $106^{*}$

Alocasia puncakborneensis S.Y. Wong \& P.C. Boyce, sp. nov. ... 75(1). 2020. p. $112^{*}$

Homalomena anthurioides S.Y. Wong, P.C. Boyce \& A. Hay, sp. nov. 75(1). 2020. p. $118^{\star}$
Homalomena pexa S.Y. Wong, P.C. Boyce \& A. Hay, sp.nov. ... 75(1). 2020. p. $120^{*}$

Monstera croatii M. Cedeño \& A. Hay, sp. nov. 75(1). 2020. p. $124^{*}$

Monstera gambensis M. Cedeño \& M.A. Blanco, sp. nov. .75(1). 2020. p. $127-129^{*}$

Vol. 75(2). 2020

Flemingia sericans Kurz, syn. nov. 75(2). 2020. p. 160

Flemingia stricta Roxb. subsp. pteropus (Baker) K.K. Khanna \& An. Kumar, syn. nov. 75(2). 2020. p. 181

Flemingia nudiflora (Haines) S.K. Gavade, Maesen \& Lekhak, comb. et stat. nov. 75(2). 2020. p. 209

Flemingia tiliacea Niyomdham, syn. nov. ...75(2). 2020. p. 209

Ophiorrhiza meenachilarensis Robi \& Anoop, sp. nov. 75(2). 2020. p. 232

Vochysia tepuiandina Huamantupa, sp. nov. 75(2). 2020. p. 238

Tachigali inca Huamantupa, H.C. Lima \& D.B.O.S. Cardoso, sp. nov. 75(2). 2020. p. $244^{*}$

Amorphophallus dactylifer Hett., syn. nov. 75(2). 2020. p. $288^{*}$ 